\title{
Correspondence
}

\section{Computed tomographic appearances of cardiac amyloidosis}

Sir,

T Sekiya et al (1984; 51: 519-22) described the usefulness of computed tomography in differentiating cardiac amyloidosis from both constrictive pericarditis and primary myocardial hypertrophy. Interestingly, they emphasised the radiological features of cardiac amyloidosis: a lower myocardial density on precontrast tomograms with diffuse thickening of the myocardium on postcontrast tomograms. These observations require some comment. Firstly, the inconspicuous diffuse thickening of the left ventricular myocardium is not (as stated in the legend of Fig. 2b) a precontrast but a postcontrast tomogram as indicated by the aortic opacification. Secondly, we completely agree with Dr Sekiya about the mean density value of precontrast computed myocardial amyloid tomograms. We have recently seen a 64 year old woman with systemic amyloidosis that was reactive to a cutaneous fistula of chronic empyema continuously fed by an oesophageal communication. Myocardial density measured by three rectangular regions of 29 pixels each (30.4 (3.4) Hounsfield units (HU)) is comparable to Dr Sekiya's findings (30.6 (3.4) $\mathrm{HU}$ ) and does not present any overlap with normal myocardial density (Figure). Thirdly, whether or not computed tomography can also differentiate cardiac amyloidosis from other forms of restrictive cardiomyopathy is debatable. It could, however, be suspected that precontrast myocardial density of cardiac haemochromatosis will be different from cardiac amyloidosis density.

Finally, whatever its exact origin-immunogenic or reactive systemic-amyloidosis in western countries is the most frequent cause of restrictive cardiomyopathy. Echocardiography delineates correctly the range of cardiomyopathies. The features of restrictive cardiomyopathy are: $(a)$ normal left and right ventricular size, $(b)$ thickened walls, $(c)$ globally reduced left ventricle, and $(d)$ left atrial enlargement (50\%) and pericardial effusion (58\%). ${ }^{1}$ A particular echocardiographic feature indicating the amyloid origin is the granular sparkling appearance of the affected cardiac walls. ${ }^{1}$ We believe therefore that echocardiography remains the cornerstone of cardiac amyloidosis diagnosis; in most cases it is the best means of excluding constrictive pericarditis. Nevertheless, the echocardiographic features of cardiac amyloidosis could sometimes be misleading in

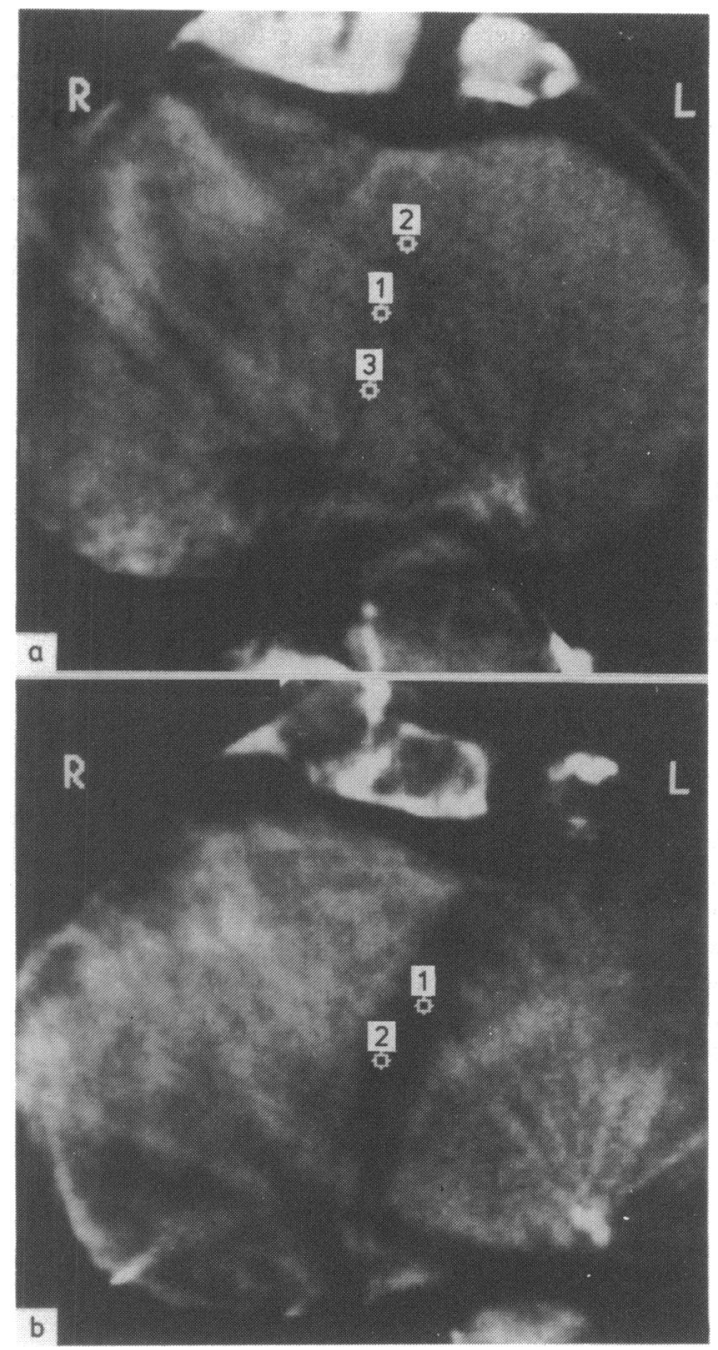

Figure Precontrast (a) and postcontrast (b) tomograms showing a low septal density before contrast injection. 1, 2, and 3 indicate the three rectangular regions of 29 pixels each. 
patients with hypertrophic cardiomyopathy. In such cases computed tomography may compete with endomyocardial biopsy in providing a correct diagnosis.

\author{
P Depelchin, ${ }^{\star}$ \\ G Kuhn, $†$ \\ Departments of ${ }^{\star}$ Medical Cardiology and \\ †Roentgenology, \\ Hopital Academique Erasme, Université Libre de \\ Bruxelles, \\ Route de Lennick 808, \\ B 1070 Bruxelles, Belgium.
}

\section{Reference}

1 Siqueira-Filho AG, Cunha CLP, Tajik AJ, Seward JB, Schattenberg TT, Giuliani ER. M-mode and twodimensional echocardiographic features in cardiac amyloidosis. Circulation 1981; 63: 188-96.

This letter was shown to the authors, who reply as follows:
Sir,

The letter by Drs Depelchin and Kuhn documents an additional case of cardiac amyloidosis, in which the computed tomographic appearances are similar to those in our paper (1984; 51: 519-22). They also correctly point out that the description of a postcontrast computed tomogram was omitted in the legend of Fig. $2 b$ in our report.

I would totally agree with their opinion that computed tomography provides important information for the diagnosis of cardiac amyloidosis but that echocardiography still remains the imaging method of choice in the screening of the disease and in the differentiation of the disease from constrictive pericarditis. Computed tomography would, however, be more useful when echocardiography is technically difficult in patients with obesity or emphysema.

Tohru Sekiya,

Department of Radiology,

Jikei University School of Medicine,

Nishi-Shinbashi,

Minato-Ku,

Tokyo 105, Japan.

\section{Use of the exercise maximal ST segment/heart rate slope in assessing the results of coronary angioplasty}

Sir,

We have read with interest the article of Silverton et al (1984; 51: 379-85), which essentially reflects our own opinion, except for a detail which we would like to point out. In fact, we are not convinced that the use of bipolar leads, especially CM5, as proposed by the authors, improves the sensitivity of the test dramatically.

We have recorded routine exercise electrocardiograms in patients before and after transluminal coronary angioplasty. All patients underwent bicycle exercise in the sitting position with monitoring leads V2, V5, and aVF. The signals were digitised and processed for averaging and artefact reduction.

In 40 patients who successfully underwent percutaneous transluminal coronary angioplasty the exercise electrocardiogram correlated well with the angiographic results: mean reduction in the degree of stenosis of $72.4 \%$ (range 84.9 to $12.5 \% ; p<0.005$ ), improvement of maximum predicted heart rate $(65.5$ (8)\% to $82(8.6) \% ; p<0.01)$, mean exercise time $(4.8$ $(1.8)$ min to $5.8(1.4)$ min; $\mathrm{p}<0.05)$, maximal ergometric load (133 W/2.8 $\mathrm{min}(18 / 1.2)$ to $152 \mathrm{~W} / 3$ $\min (11 / 1.6) ; p<0.05)$, and maximal rate pressure product $(16.592(128)$ to $25.807(161) ; \mathrm{p}<0.001)$. The maximal ST depression at postangiography ergometry fell from $2.8(1.1) \mathrm{mm}$ to $0.38(0.06) \mathrm{mm}(\mathrm{p}<0.01)$. In 10 patients in whom transluminal coronary angioplasty was unsuccessful we failed to observe any significant change in the exercise electrocardiogram after operation.

While we agree with Silverton et al that exercise testing is of value in the follow up of patients after transluminal coronary angioplasty, we consider that the same good correlations between stenosis reduction and improvement of the exercise electrocardiogram can be obtained with unipolar conventional (V2, V5, aVF) leads.

Leo Finci, Jean Jacques Goy, Raymond Maendly, Milan Grbic, Ulrich Sigwart, Division of Cardiology, Department of Internal Medicine, Centre Hospitalier Universitaire Vaudois, CH-1011 Lausanne, Switzerland. 